\title{
Growth of the entire or meromorphic solutions of Differential- difference equations
}

\author{
RAJ SHREE DHAR \\ Higher Education Department, J and K Government \\ Jammu and Kashmir,INDIA \\ dhar.rajshree@jk.gov.in
}

November 24, 2018

\begin{abstract}
In this paper, we study the entire or meromorphic solutions for differentialdifference equations in $\mathrm{f}(\mathrm{z})$, its shifts, its derivatives and derivatives of its shifts. and study some Hayman's results for differential- difference polynomials .
\end{abstract}

Mathematics Subject Classification:

2010 : Primary 30D35. Secondary 30D30,39A05.

Keywords : Entire and Meromorphic functions, Differential- difference polynomial, Shared value, Nevanlinna theory.

\section{INTRODUCTION AND MAIN RESULTS:}

It is assumed that the reader is familiar with the basic concepts of Nevanlinna Theory, see e.g. ([1],[2]), such as the characteristic function $\mathrm{T}(\mathrm{r}, \mathrm{f})$, proximity function $\mathrm{m}(\mathrm{r}, \mathrm{f})$, counting function $\mathrm{N}(\mathrm{r}, \mathrm{f})$ and so on. In addition, $\mathrm{S}(\mathrm{r}, \mathrm{f})$ denotes any quantity that satisfies the condition that $S(r, f)=o(T(r, f))$ as $r$ tends to infinity outside of a possible exceptional set of finite logarithimic measure. In the sequel, a meromorphic function a(z)is called a small function with respect to $\mathrm{f}$ if and only if $\mathrm{T}[\mathrm{r}, \mathrm{a}(\mathrm{z})]=\mathrm{o}(\mathrm{T}(\mathrm{r}, \mathrm{f}))$ as $\mathrm{r}$ tends to infinity outside of a possible exceptional set of finite logarithimic measure. We denote by $\mathrm{S}(\mathrm{f})$, the family of all such small meromorphic functions.

We say that two meromorphic functions $\mathrm{f}$ and $\mathrm{g}$ share the value a (belonging to extended complex plane) CM (IM)

provided that

$$
f(z) \equiv a
$$


if and only if

$$
g(z) \equiv a
$$

counting multiplicity (ignoring multiplicity).

\section{DEFINITION 1 :}

Let $\mathrm{c}$ be a non-zero complex costant then for a meromorphic function $\mathrm{f}(\mathrm{z})$, we define its shift by $\mathrm{f}(\mathrm{z}+\mathrm{c})$ and its difference operator by

$$
\begin{gathered}
\Delta_{c} f(z)=f(z+c)-f(z), \\
\Delta_{m c} f(z)=f(z+m c)-f(z),
\end{gathered}
$$

where $\mathrm{m}$ is a positive integer

$$
\Delta_{c}^{n} f(z)=\Delta_{c}^{n-1}\left(\Delta_{c} f(z)\right)
$$

$\mathrm{n} \in \mathbf{N}, n \geq 2$,

$$
=\sum_{k=0}^{n} \frac{(-1)^{k} \cdot n !}{k ! .(n-k) !} f(z+\overline{n-k} \cdot c) .
$$

In particular,

$$
\Delta_{c}^{n} f(z)=\Delta^{n} f(z)
$$

for $\mathrm{c}=1$.

We define Differential - difference Monomial as

$$
M[f]=\prod_{i=0}^{k} \prod_{j=0}^{m}\left[f^{(j)}\left(z+c_{i j}\right)\right]^{n} i j
$$

where $c_{i j}$ are complex constants, and $n_{i j}$ are natural numbers , $\mathrm{i}=0,1, \ldots$ , $\mathrm{k}$ and $\mathrm{j}=0,1, \ldots, \mathrm{m}$.

Then the degree of $\mathbf{M}[\mathbf{f}]$ will be the sum of all the powers in the product on the right hand side. Let us define the weight of $\mathbf{M}[\mathbf{f}]$ as $\Gamma_{M}=$ sum of powers of $f+2$.sum of powers of $f^{\prime}+3$. sum of powers of $f^{\prime \prime}+\ldots$

\section{DEFINITION 2 :}

Let

$$
M_{1}[f], M_{2}[f], \ldots
$$

denote the distinct monomials in $\mathrm{f}$, and

$$
a_{1}(z), a_{2}(z), \ldots
$$

be the small meromorphic functions including complex numbers then

$$
P[f]=P[z, f]=\sum_{j \in \Delta} a_{j}(z) \cdot M_{j}[f]
$$


where $\Delta$ is a finite set of multi- indices, $a_{j}(z)$ are small functions of $\mathrm{f}, M_{j}[f]$ are differential- difference monomials,

will be called a differential- difference polynomial in $\mathrm{f}$, which is a finite sum of products of $f$, derivatives of $f$, their shifts, and derivatives of its shifts.

Let us define the total degree $\mathrm{d}$ and weight $\Gamma$ of $\mathrm{P}[\mathrm{z}, \mathrm{f}]$ in $\mathrm{f}$ as

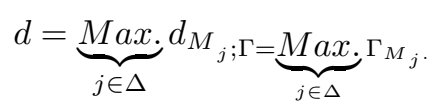

If all the terms in the summation of $\mathrm{P}[\mathrm{f}]$ have same degrees, then $\mathrm{P}[\mathrm{f}]$ is known as homogeneous differential- difference polynomial. Usually, we take $\mathrm{P}[\mathrm{f}]$ such that $\mathrm{T}(\mathrm{r}, \mathrm{P}) \neq S(r, f)$.

A finite value a is called the Picard exceptional value of $f$, if $f-a$ has no zeros. The Picard theorem shows that a transcendental entire function has at most one Picard exceptional value, a transcendental meromorphic functions has at most two picard exceptional values.

\section{SECTION 1:}

W. K.Hayman[1] proved the following theorem:

THEOREM A[1]: If $\mathrm{f}(\mathrm{z})$ is meromorphic and transcendental in the plane and has only a finite number of zeros and poles, then every non-constant function

$$
\varphi(z)=\Sigma a_{j}(z) \cdot f^{j}(z), j=0, \ldots, l
$$

assumes every finite value except possibly zero infinitely often.

We will consider the differential- difference polynomial as in definition 2 and prove Theorem A for such polynomials as the following results:

\section{MAIN RESULTS:}

THEOREM 1.1: Let $\mathrm{f}$ be a transcendental entire function with finite order and as in definition $2, \mathrm{P}[\mathrm{f}]$ be a differential- difference polynomial of degree $\mathrm{d}$ defined as

$$
P[f]=\sum_{j \in \Delta} a_{j}(z) \cdot M_{j}[f] ; T(r, P[f]) \neq S(r, f),
$$

where $\Delta$ is a finite set of multi- indices, $a_{j}(z)$ are small functions of $\mathrm{f}, M_{j}[f]$ are differential- difference monomials, then $\mathrm{P}[\mathrm{f}]=\mathrm{a}(\mathrm{z})(\mathrm{a}(\mathrm{z})=$ small function or complex value, $\mathrm{a}(\mathrm{z}) \neq 0, \infty)$ has infinitely many solutions provided $\mathrm{N}(\mathrm{r}, 0, \mathrm{f})$ $=\mathrm{S}(\mathrm{r}, \mathrm{f})$. 
THEOREM 1.2: Let $\mathrm{f}$ be a transcendental meromorphic function with finite order and as in definition $2, \mathrm{P}[\mathrm{f}]$ be a differential- difference polynomial of degree d defined as

$$
P[f]=\sum_{j \in \Delta} a_{j}(z) . M_{j}[f] ; T(r, P[f]) \neq S(r, f),
$$

where $\Delta$ is a finite set of multi- indices, $a_{j}(z)$ are small functions of $\mathrm{f}, M_{j}[f]$ are differential- difference monomials, then $\mathrm{P}[\mathrm{f}]=\mathrm{a}(\mathrm{z})(\mathrm{a}(\mathrm{z})=$ small function or complex value, $\mathrm{a}(\mathrm{z}) \neq 0, \infty)$ has infinitely many solutions provided $\mathrm{N}(\mathrm{r}, 0, \mathrm{f})$ $+\mathrm{N}(\mathrm{r}, \mathrm{f})=\mathrm{S}(\mathrm{r}, \mathrm{f})$.

The classical problem of value distributions of differential polynomials is Hayman conjecture [3],i.e. if $\mathrm{f}$ is a transcendental meromorphic function and $\mathrm{n} \in$ $\mathrm{N}$, then $\mathrm{f}^{n} f^{\prime}$ takes every finite non-zero value infinitely often which means that the Picard exceptional value of $\mathrm{f}^{n} f^{\prime}$ may only be zero. This conjecture has been proved by many authors. e.g., Hayman [3] proved that if $\mathrm{f}$ is a transcendental meromorphic function and $\mathrm{n} \geq 3$, then $\mathrm{f}^{n} f^{\prime}$ takes every finite non-zero complex value infinitely often. The case $n=2$ was proved by Mues[4], and Bergweiler et. al[5] proved the case for $n=1$.

Then many authors started to investigate the uniqueness of meromorphic functions sharing values with their shifts / q-shifts or difference operators see e.g. ([6]-[9]).

We shall prove the above conjecture for general differential difference polynomials with some condition on power of $f$.

THEOREM 1.3: Let $\mathrm{f}$ be a transcendental meromorphic function with finite order and as in definition $2, \mathrm{P}[\mathrm{f}]$ be a differential- difference polynomial of degree $\mathrm{d}$ and weight $\Gamma$ defined as

$$
P[f]=P[z, f]=\sum_{j \in \Delta} a_{j}(z) \cdot M_{j}[f]
$$

$T(r, P[f]) \neq \mathrm{S}(\mathrm{r}, \mathrm{f})$, where $\Delta$ is a finite set of multi- indices, $a_{j}(z)$ are small functions of $\mathrm{f}, M_{j}[f]$ are differential- difference monomials,

then $\mathrm{f}^{l}(f-1) P[f]-a(z), a(z) \neq 0, \infty$ has infinitely many zeros provided $\mathrm{l}>$ $2(\Gamma+1)$.

For the proof of the results we need the following lemmas:

LEMMA 1 [7]: Let $\mathrm{f}$ be a non- constant meromorphic function of finite order and c be a non- zero complex constant, then

$$
m\left(r, \frac{f(z+c)}{f(z)}\right)=\mathrm{S}(\mathrm{r}, \mathrm{f})
$$


for all $\mathrm{r}$ outside a possible exceptional set of finite logarithmic measure.

LEMMA 2[7]: Let c be a non-zero complex constant, and let $\mathrm{f}$ be a meromorphic function of finite order then

$$
\begin{aligned}
& T(r, f(z+c))=T(r, f)+S(r, f) \\
& N(r, f(z+c))=N(r, f)+S(r, f) \\
& N(r, 0, f(z+c))=N(r, 0, f)+S(r, f)
\end{aligned}
$$

LEMMA 3(Clunie type lemma $[6])$ : Let $\mathrm{f}(\mathrm{z})$ be a non- constant meromorphic function of finite order such that

$$
f^{n} P[z, f]=Q[z, f],
$$

where $\mathrm{P}[\mathrm{z}, \mathrm{f}], \mathrm{Q}[\mathrm{z}, \mathrm{f}]$ are differential-difference polynomials in $\mathrm{f}$. If the degree of $\mathrm{Q}[\mathrm{z}, \mathrm{f}]$ as a polynomial in $\mathrm{f}$ and its shifts is at most $\mathrm{n}$, then

$$
m(r, P[z, f])=S(r, f) .
$$

LEMMA 4([11]): Let $\mathrm{f}$ be a nonconstant meromorphic function. If $\mathrm{Q}[\mathrm{f}]$ is a differential polynomial in $\mathrm{f}$ with arbitrary meromorphic coefficients, then

( i) $\mathrm{m}(\mathrm{r}, \mathrm{Q}[\mathrm{f}]) \leq \gamma_{Q} m(r, f)+\mathrm{S}(\mathrm{r}, \mathrm{f})$

(ii) $\mathrm{N}(\mathrm{r}, \mathrm{Q}[\mathrm{f}]) \leq \Gamma_{Q} N(r, f)+S(r, f)$

Remark: We can obtain Lemma 4 for differential difference polynomials in $\mathrm{f}$ of finite order using lemma 2 and definition of weight as in definition 2 .

PROOF OF THEOREM 1.1:

Let $\mathrm{P}[\mathrm{f}]=\mathrm{a}(\mathrm{z}), \mathrm{a}(\mathrm{z}) \neq 0, \infty$ has finitely many solutions.

. Then we get by using Lemma 2, Lemma 3 and given condition:

$$
\begin{aligned}
& \qquad T(r, P[f])=T\left(r, \sum_{j \in \Delta} a_{j}(z) \cdot M_{j}[f]\right) \\
& =\mathrm{T}\left(\mathrm{r}, \mathrm{f}^{d}\left[a_{1} \frac{M_{1}}{f^{d}}+a_{2} \frac{M_{2}}{f^{d}}+\ldots+a_{n} \frac{M_{n}}{f^{d}}\right]\right) \\
& \geq \mathrm{d} \mathrm{T}(\mathrm{r}, \mathrm{f})-\mathrm{T}\left(\mathrm{r},\left[\mathrm{a}_{1} \frac{M_{1}}{f^{d}}+a_{2} \frac{M_{2}}{f^{d}}+\ldots+a_{n} \frac{M_{n}}{f^{d}}\right]\right) \\
& \text { But } \mathrm{T}\left(\mathrm{r},\left[\mathrm{a}_{1} \frac{M_{1}}{f^{d}}+a_{2} \frac{M_{2}}{f^{d}}+\ldots+a_{n} \frac{M_{n}}{f^{d}}\right]\right) \\
& \leq \mathrm{T}\left(\mathrm{r}, \frac{M_{1}}{f^{d}}\right)+\mathrm{T}\left(\mathrm{r}, \frac{M_{2}}{f^{d}}\right)+\ldots+\mathrm{T}\left(\mathrm{r}, \frac{M_{n}}{f^{d}}\right)+\mathrm{S}(\mathrm{r}, \mathrm{f})
\end{aligned}
$$




$$
\begin{aligned}
& =\mathrm{N}\left(\mathrm{r}, \frac{M_{1}}{f^{d}}\right)+\mathrm{N}\left(\mathrm{r}, \frac{M_{2}}{f^{d}}\right)+\ldots+\mathrm{N}\left(\mathrm{r}, \frac{M_{n}}{f^{d}}\right)+\mathrm{S}(\mathrm{r}, \mathrm{f}) \\
& =\mathrm{S}(\mathrm{r}, \mathrm{f})
\end{aligned}
$$

Therefore, we have

$$
\begin{gathered}
\mathrm{T}(\mathrm{r}, \mathrm{P}[\mathrm{f}]) \\
\geq \mathrm{d} \mathrm{T}(\mathrm{r}, \mathrm{f})+\mathrm{S}(\mathrm{r}, \mathrm{f})
\end{gathered}
$$

Since $f$ is entire, therefore, by using Nevanlinna's second main theorem, we get

$$
\begin{aligned}
& \mathrm{d} \mathrm{T}(\mathrm{r}, \mathrm{f}) \leq \mathrm{T}(\mathrm{r}, \mathrm{P}[\mathrm{f}]) \leq \bar{N}\left(r, \frac{1}{P[f]}\right)+\bar{N}(r, P[f])+\bar{N}\left(r, \frac{1}{P[f]-a(z)}\right)+\mathrm{S}(\mathrm{r}, \mathrm{f}) \\
& =\mathrm{S}(\mathrm{r}, \mathrm{f})
\end{aligned}
$$

which is a contradiction as $d \geq 1$. Thus our supposition is wrong and hence, $\mathrm{P}[\mathrm{f}]=\mathrm{a}(\mathrm{z})$ (small function or complex value), $\mathrm{a}(\mathrm{z}) \neq 0, \infty$ has infinitely many solutions.

\section{PROOF OF THEOREM 1.2:}

Let $\mathrm{P}[\mathrm{f}]=\mathrm{a}(\mathrm{z})$ (small function or complex value), $\mathrm{a}(\mathrm{z}) \neq 0, \infty$ has finitely many solutions.

Then we get by using Lemma 2, Lemma 3 and given condition:

$$
\begin{aligned}
& \qquad T(r, P[f])=T\left(r, \sum_{j \in \Delta} a_{j}(z) \cdot M_{j}[f]\right) \\
& =\mathrm{T}\left(\mathrm{r}, \mathrm{f}^{d}\left[a_{1} \frac{M_{1}}{f^{d}}+a_{2} \frac{M_{2}}{f^{d}}+\ldots+a_{n} \frac{M_{n}}{f^{d}}\right]\right) \\
& \geq \mathrm{d} \mathrm{T}(\mathrm{r}, \mathrm{f})-\mathrm{T}\left(\mathrm{r},\left[\mathrm{a}_{1} \frac{M_{1}}{f^{d}}+a_{2} \frac{M_{2}}{f^{d}}+\ldots+a_{n} \frac{M_{n}}{f^{d}}\right]\right) \\
& \text { But } \mathrm{T}\left(\mathrm{r},\left[\mathrm{a}_{1} \frac{M_{1}}{f^{d}}+a_{2} \frac{M_{2}}{f^{d}}+\ldots+a_{n} \frac{M_{n}}{f^{d}}\right]\right) \\
& \leq \mathrm{T}\left(\mathrm{r}, \frac{M_{1}}{f^{d}}\right)+\mathrm{T}\left(\mathrm{r}, \frac{M_{2}}{f^{d}}\right)+\ldots+\mathrm{T}\left(\mathrm{r}, \frac{M_{n}}{f^{d}}\right)+\mathrm{S}(\mathrm{r}, \mathrm{f}) \\
& =\mathrm{N}\left(\mathrm{r}, \frac{M_{1}}{f^{d}}\right)+\mathrm{N}\left(\mathrm{r}, \frac{M_{2}}{f^{d}}\right)+\ldots+\mathrm{N}\left(\mathrm{r}, \frac{M_{n}}{f^{d}}\right)+\mathrm{S}(\mathrm{r}, \mathrm{f}) \\
& =\mathrm{S}(\mathrm{r}, \mathrm{f})
\end{aligned}
$$

Therefore, we have

$\mathrm{T}(\mathrm{r}, \mathrm{P}[\mathrm{f}])$

$\geq \mathrm{d} \mathrm{T}(\mathrm{r}, \mathrm{f})+\mathrm{S}(\mathrm{r}, \mathrm{f})$ 
Since $f$ is meromorphic and by using $N(r, 0, f)+N(r, f)=S(r, f)$ and Nevanlinna's second main theorem, we get

$$
\begin{aligned}
& \mathrm{d} \mathrm{T}(\mathrm{r}, \mathrm{f}) \leq \mathrm{T}(\mathrm{r}, \mathrm{P}[\mathrm{f}]) \leq \bar{N}\left(r, \frac{1}{P[f]}\right)+\bar{N}(r, P[f])+\bar{N}\left(r, \frac{1}{P[f]-a(z)}\right)+\mathrm{S}(\mathrm{r}, \mathrm{f}) \\
& =\mathrm{S}(\mathrm{r}, \mathrm{f})
\end{aligned}
$$

which is a contradiction as $d \geq 1$. Thus our supposition is wrong and hence, $\mathrm{P}[\mathrm{f}]=\mathrm{a}(\mathrm{z})$ (small function or complex value), $\mathrm{a}(\mathrm{z}) \neq 0, \infty$ has infinitely many solutions.

\section{PROOF OF THEOREM 1.3:}

Let $\mathrm{G}[\mathrm{z}]=\mathrm{f}^{l}(f-1) P[z, f]$ where $\mathrm{f}$ is a meromorphic function and suppose $\mathrm{G}[\mathrm{z}]-\mathrm{a}(\mathrm{z}), \mathrm{a}(\mathrm{z}) \neq 0, \infty$ has finitely many zeros. Then we get by using Lemma 4 for differential difference polynomials

$$
\begin{aligned}
& \mathrm{T}(\mathrm{r}, \mathrm{G}[\mathrm{z}])=\mathrm{T}\left(\mathrm{r}, \mathrm{f}^{l}(f-1) \sum_{j \in \Delta} a_{j}(z) \cdot M_{j}[f]\right) \\
& \geq(\mathrm{l}+1) \mathrm{T}(\mathrm{r}, \mathrm{f})-\Gamma T(r, f)
\end{aligned}
$$

Therefore, we have

$$
\begin{aligned}
& \mathrm{T}(\mathrm{r}, \mathrm{G}[\mathrm{z}]) \\
& \geq(1+1-\Gamma) T(r, f)+S(r, f)
\end{aligned}
$$

Since $\mathrm{f}$ is meromorphic, therefore, by using Nevanlinna's second main theorem and lemma 4 for differential difference polynomials, we get

$$
\begin{aligned}
& (1+1-\Gamma) T(r, f) \leq \mathrm{T}(\mathrm{r}, \mathrm{G}[\mathrm{z}]) \leq \bar{N}\left(r, \frac{1}{G(z)}\right)+\bar{N}(r, G(z))+\bar{N}\left(r, \frac{1}{G(z)-a(z)}\right) \\
+ & \mathrm{S}(\mathrm{r}, \mathrm{G}) \\
& =\bar{N}\left(r, \frac{1}{G(z)}\right)+\bar{N}(r, G(z))+S(r, f) \\
& \leq(\Gamma+2) T(r, f)+\bar{N}(r, f)+S(r, f) \\
& =(\Gamma+3) T(r, f)+S(r, f)
\end{aligned}
$$

So we get

$$
\mathrm{l} \mathrm{T}(\mathrm{r}, \mathrm{f}) \leq 2(\Gamma+1) T(r, f)+\mathrm{S}(\mathrm{r}, \mathrm{f})
$$

which is a contradiction as $l>2(\Gamma+1)$. Thus our supposition is wrong and hence the result. 


\section{SECTION 2}

Hayman[3] proved that a differential polynomial $\mathrm{f}^{n}+a f^{\prime}-b$ with constant coefficients $\mathrm{a}, \mathrm{b}$ admits infinitely many zeros provided that $\mathrm{f}$ is transcendental entire and $n \geq 3$. K. Liu and I. Laine in 2010 proved that:

THEOREM $B[12]$ : Let $f$ be a transcendental entire function of finite ordernot of period $\mathrm{c}$, then for small non-zero function $\mathrm{s}(\mathrm{z})$, the difference polynomial $\mathrm{f}^{n}+f(z+c)-f(z)-s(z)$ has infinitely many zeros in the complex plane provided that $\mathrm{n} \geq 3$.

We prove the above results for general difference polynomials ( shifts and difference operators as in definition 1 are part of these) as following:

THEOREM 2.1: Let $\mathrm{f}$ be a transcendental entire function with finite order and as in definition $2, \mathrm{P}[\mathrm{f}]$ be a linear difference polynomial defined as $P[f]=c_{0} f(z)+c_{1} f(z+c)+c_{2} f(z+2 c)+\ldots+c_{n} f(z+n c) ; T(r, P[f]) \neq \mathrm{S}(\mathrm{r}, \mathrm{f})$, where $\mathrm{c} \neq 0$ and $\mathrm{c}_{j}, j=0,1, \ldots, n$, are complex constants then $\mathrm{f}^{l}+P[f]-$ $a(z), a(z) \neq 0, \infty$ has infinitely many zeros provided $\mathrm{l}>2 \mathrm{n}+1$.

THEOREM 2.2: Let $\mathrm{f}$ be a transcendental meromorphic function with finite order and as in definition 2, $\mathrm{P}[\mathrm{f}]$ be a linear difference polynomial defined as $P[f]=c_{0} f(z)+c_{1} f(z+c)+c_{2} f(z+2 c)+\ldots+c_{n} f(z+n c) ; T(r, P[f]) \neq \mathrm{S}(\mathrm{r}, \mathrm{f})$, where $\mathrm{c} \neq 0$ and $\mathrm{c}_{j}, j=0,1, \ldots, n$, are complex constants then $\mathrm{f}^{l}+P[f]-$ $a(z), a(z) \neq 0, \infty$ has infinitely many zeros provided $1>3 \mathrm{n}+3$.

\section{PROOF OF THEOREM 2.1:}

Let $\mathrm{G}[\mathrm{z}]=\mathrm{f}^{l}+P[f]$ where $\mathrm{f}$ is an entire function and suppose $\mathrm{G}[\mathrm{z}]-\mathrm{a}(\mathrm{z}), \mathrm{a}(\mathrm{z}) \neq$ $0, \infty$ has finitely many zeros. Then we get by using Lemma 1 and Lemma 2

$$
\begin{aligned}
& \mathrm{T}(\mathrm{r}, \mathrm{G}[\mathrm{z}])=\mathrm{T}\left(\mathrm{r}, \mathrm{f}^{l}+\left[c_{0} f(z)+c_{1} f(z+c)+c_{2} f(z+2 c)+\ldots+c_{n} f(z+n c)\right]\right) \\
& \geq(\mathrm{l}+1) \mathrm{T}(\mathrm{r}, \mathrm{f})-\mathrm{T}\left(\mathrm{r},\left[c_{1} f(z+c)+c_{2} f(z+2 c)+\ldots+c_{n} f(z+n c)\right]\right)
\end{aligned}
$$

Therefore, we have

$$
\begin{gathered}
\mathrm{T}(\mathrm{r}, \mathrm{G}[\mathrm{z}]) \\
\geq(\mathrm{l}-\mathrm{n}+1) \mathrm{T}(\mathrm{r}, \mathrm{f})+\mathrm{S}(\mathrm{r}, \mathrm{f})
\end{gathered}
$$

Since $\mathrm{f}$ is entire, therefore, by using Nevanlinna's second main theorem, we get

$$
\begin{aligned}
& {[\mathrm{l}+1-\mathrm{n}] \mathrm{T}(\mathrm{r}, \mathrm{f}) \leq \mathrm{T}(\mathrm{r}, \mathrm{G}[\mathrm{z}]) \leq \bar{N}\left(r, \frac{1}{G(z)}\right)+\bar{N}\left(r, \frac{1}{G(z)-a(z)}\right)+\mathrm{S}(\mathrm{r}, \mathrm{G})} \\
& =\bar{N}\left(r, \frac{1}{G(z)}\right)+\mathrm{S}(\mathrm{r}, \mathrm{f})
\end{aligned}
$$




$$
\begin{aligned}
& \leq(\mathrm{n}+2) \mathrm{N}(\mathrm{r}, 0, \mathrm{f})+S(r, f) \\
& \leq(\mathrm{n}+2) \mathrm{T}(\mathrm{r}, \mathrm{f})+S(r, f)
\end{aligned}
$$

So we get

$$
\mathrm{l} \mathrm{T}(\mathrm{r}, \mathrm{f}) \leq(2 \mathrm{n}+1) \mathrm{T}(\mathrm{r}, \mathrm{f})+S(r, f)
$$

which is a contradiction as $\mathrm{l}>2 \mathrm{n}+1$. Thus our supposition is wrong and hence, $f^{l} P[f]-a(z), a(z) \neq 0, \infty$ has infinitely many zeros.

\section{PROOF OF THEOREM 2.2:}

Let $\mathrm{G}[\mathrm{z}]=\mathrm{f}^{l}+P[f]$ where $\mathrm{f}$ is a meromorphic function and suppose $\mathrm{G}[\mathrm{z}]-\mathrm{a}(\mathrm{z})$, $\mathrm{a}(\mathrm{z}) \neq 0, \infty$ has finitely many zeros. Then we have

$$
\begin{aligned}
& \mathrm{T}(\mathrm{r}, \mathrm{G}[\mathrm{z}])=\mathrm{T}\left(\mathrm{r}, \mathrm{f}^{l}+\left[c_{0} f(z)+c_{1} f(z+c)+c_{2} f(z+2 c)+\ldots+c_{n} f(z+n c)\right]\right) \\
& \geq(\mathrm{l}+1) \mathrm{T}(\mathrm{r}, \mathrm{f})-\mathrm{T}\left(\mathrm{r},\left[\mathrm{c}_{1} f(z+c)+c_{2} f(z+2 c)+\ldots+c_{n} f(z+n c)\right]\right)
\end{aligned}
$$

Therefore, we have

$$
\begin{aligned}
& \mathrm{T}(\mathrm{r}, \mathrm{G}[\mathrm{z}]) \\
& \geq(\mathrm{l}-\mathrm{n}+1) \mathrm{T}(\mathrm{r}, \mathrm{f})+\mathrm{S}(\mathrm{r}, \mathrm{f})
\end{aligned}
$$

Since $\mathrm{f}$ is meromorphic, therefore, by using Nevanlinna's second main theorem and lemma, we get

$$
\begin{aligned}
& {[\mathrm{l}-\mathrm{n}+1] \mathrm{T}(\mathrm{r}, \mathrm{f}) \leq \mathrm{T}(\mathrm{r}, \mathrm{G}[\mathrm{z}]) \leq \bar{N}\left(r, \frac{1}{G(z)}\right)+\bar{N}(r, G(z))+\bar{N}\left(r, \frac{1}{G(z)-a(z)}\right) } \\
+ & \mathrm{S}(\mathrm{r}, \mathrm{G}) \\
& =\bar{N}\left(r, \frac{1}{G(z)}\right)+\bar{N}(r, G(z))+S(r, f) \\
& \leq(2 \mathrm{n}+4) \mathrm{T}(\mathrm{r}, \mathrm{f})+S(r, f)
\end{aligned}
$$

So we get

$$
\mathrm{l} \mathrm{T}(\mathrm{r}, \mathrm{f}) \leq(3 \mathrm{n}+3) \mathrm{T}(\mathrm{r}, \mathrm{f})+S(r, f)
$$

which is a contradiction as $\mathrm{l}>3 \mathrm{n}+3$. Thus our supposition is wrong and hence, $\mathrm{f}^{l}+P[f]-a(z), a(z) \neq 0, \infty$ has infinitely many zeros.

\section{REFERENCES:}

1. Hayman, W.K.: Meromorphic functions- Clarenden Press, Oxford, 1964. 
2. Yi, H.X., Yang, C.C.: Uniqueness Theory of Meromorphic Functions- Kluwer Academic, Dordrecht (2003).[Chinese original: Science Press, Beijing (1995)].

3. Hayman, W.K.: Picard values of meromorphic functions and their derivativesAnn. of Math. (2), 70 (1959), pp.9-42.

4. Mues E, ber ein: Problem von Hayman- Math. Z. 164 (1979), pp.239259.

5. Bergweiler, W. and Eremenko, A.: On the singularities of the inverse to a meromorphic function of finite order- Revista Matemtica Iberoamericana, 11 (1995), pp.355-373.

6. Laine, I., Yang, C.C.: Value distribution and uniqueness of difference polynomials -Proc. Japan Acad. Ser. A, 83(2007), pp.148-151.

7. Zhang, J. and Korhonen,R.: On the Nevanlinna characteristic of $\mathrm{f}(\mathrm{qz})$ and its applications- Journal of Mathematical Anal. and Appl., vol. 369, no. 2, 2010, pp.537-544.

8. Barnett,D., Halburd,R.J., Korhonen, Morgan: Applications of Nevanlinna theory to q-difference equations- Proc. Roy. Soc. Edinburgh Sect. A 137(2007), pp.457-474.

9. Liu, K., Liu, X., Cao, T.B. : Value distribution of the difference operator, Advances in Difference equations, Vol.2011, Art ID 234215,12 pages.

10. Yang, C.C., Hua, X.H.: Uniqueness and value sharing of meromorphic functions, Ann. Acad.Sci. Fenn. Math. 22(1997), pp.395-406.

11. Doeringer, W.: Exceptional values of Differential Polynomials, Pacific J. of Math., 98(no. 1) 1982, pp.55-62.

12. Liu, K., Laine, I.: A note on value distributionof difference polynomials, Bull. Aust. Math. Soc. 81(2010), 353-360. 